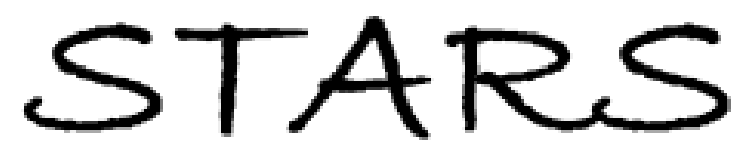

University of Central Florida

STARS

$1-1-2006$

\title{
Linear to axial or radial polarization conversion using a liquid crystal gel
}

Hongwen Ren

University of Central Florida

Yi-Hsin Lin

University of Central Florida

Shin-Tson Wu

University of Central Florida

Find similar works at: https://stars.library.ucf.edu/facultybib2000

University of Central Florida Libraries http://library.ucf.edu

This Article is brought to you for free and open access by the Faculty Bibliography at STARS. It has been accepted for inclusion in Faculty Bibliography 2000 s by an authorized administrator of STARS. For more information, please contact STARS@ucf.edu.

\section{Recommended Citation}

Ren, Hongwen; Lin, Yi-Hsin; and Wu, Shin-Tson, "Linear to axial or radial polarization conversion using a liquid crystal gel" (2006). Faculty Bibliography 2000s. 6500.

https://stars.library.ucf.edu/facultybib2000/6500

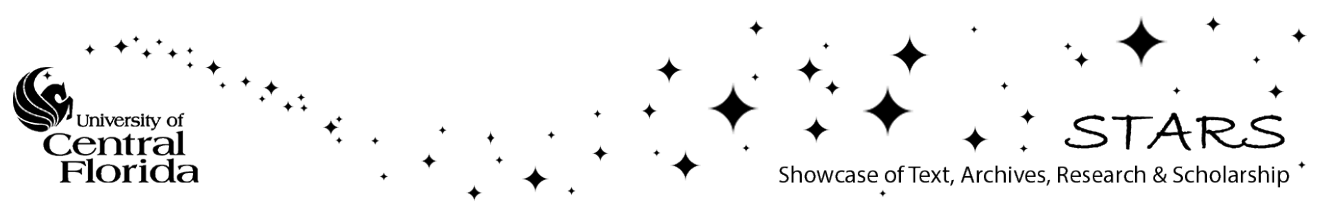




\section{Linear to axial or radial polarization conversion using a liquid crystal gel}

Cite as: Appl. Phys. Lett. 89, 051114 (2006); https://doi.org/10.1063/1.2335589

Submitted: 01 May 2006 . Accepted: 24 June 2006 . Published Online: 03 August 2006

Hongwen Ren, Yi-Hsin Lin, and Shin-Tson Wu

ARTICLES YOU MAY BE INTERESTED IN

Radially polarized optical vortex converter created by femtosecond laser nanostructuring of glass

Applied Physics Letters 98, 201101 (2011); https://doi.org/10.1063/1.3590716

The formation of laser beams with pure azimuthal or radial polarization

Applied Physics Letters 77, 3322 (2000); https://doi.org/10.1063/1.1327271

Generation of arbitrary vector beams with liquid crystal polarization converters and vectorphotoaligned q-plates

Applied Physics Letters 107, 241102 (2015); https://doi.org/10.1063/1.4937592

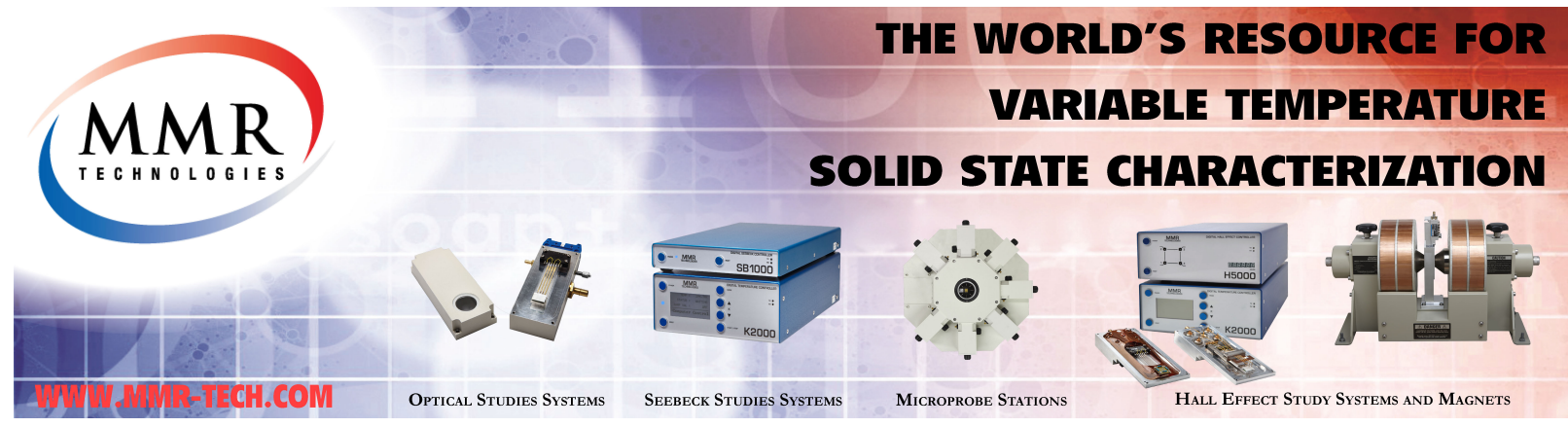




\title{
Linear to axial or radial polarization conversion using a liquid crystal gel
}

\author{
Hongwen Ren, Yi-Hsin Lin, and Shin-Tson $\mathrm{Wu}^{\mathrm{a})}$ \\ College of Optics and Photonics, University of Central Florida, Orlando, Florida 32816
}

(Received 1 May 2006; accepted 24 June 2006; published online 3 August 2006)

\begin{abstract}
Two types of liquid crystal (LC) gels with radial or twisted-radial molecular orientation are fabricated using a radial electric field generated by an indium-tin-oxide hole electrode in the bottom substrate. If the top substrate is not buffed, the radial-type LC gel is formed which can convert linearly polarized light into axially polarized light. On the other hand, if the top substrate is homogeneously buffed, then a twisted-radial LC gel is produced which can convert linearly polarized light into radially polarized light. These polarization converters are useful for diffractive optics and optical imaging systems. (C) 2006 American Institute of Physics.
\end{abstract}

[DOI: $10.1063 / 1.2335589]$

A polarization converter is an optical device which can convert randomly polarized light into linearly polarized light or convert a linearly polarized light into circular, linear, axial, or radial polarized light. It has been widely used in diffractive optics and biological tissue imaging systems. Several optical devices can be used to generate a linearly, circularly, or elliptically polarized light, but only few devices can produce axial or radial polarization. An axial polarization converter can be used as a polarization axis finder ${ }^{1}$ and a radial polarization converter can be used for analyzing biological tissue or polarizing material. ${ }^{2}$ Moreover, an axial polarizer can function as a phase modulator or tunable focus lens for a radially polarized laser beam. ${ }^{3}$

Liquid crystal (LC) is a strong contender as a polarization converter because its directors can be reoriented by an electric field. Three approaches have been proposed to produce axial, radial, or azimuthal polarization, depending on the specific LC configurations. The first approach uses a homogeneously aligned LC spatial light modulator to create the axial polarizer. ${ }^{2}$ However, the operation is rather complicated and the device is costly. The second method is to control LC directors with concentrically circular rubbing. ${ }^{4}$ The third one is to mechanically shear a polymer network liquid crystal with a tangential component. ${ }^{3}$ Some common concerns of the above mentioned approaches are complicated fabrication processes, defects, and limited aperture sizes.

In this letter, we demonstrate two types of LC gels with radial or twisted-radial molecular orientation using a radial electric field generated by a hole electrode in the bottom substrate. If the top substrate is not buffed, the radial-type LC gel is formed which can convert linearly polarized light into axially polarized light. If the top substrate is homogeneously buffed, then a twisted-radial LC gel is produced, which can convert linearly polarized light into radially polarized light. The major advantages of our devices are simple fabrication processes with capabilities for large sizes. Based on this approach, various LC converters including a polarization converter array can be fabricated.

During experimentation, we use a radial electric field to control the LC orientation. Figure 1 shows the side view of the device structure and how the electric field is generated. The LC cell consists of a thin $(\sim 0.1 \mathrm{~mm})$ top glass substrate

\footnotetext{
${ }^{a)}$ Electronic mail: swu@ mail.ucf.edu
}

which does not have any electrodes and a bottom glass substrate whose indium-tin-oxide (ITO) electrode is etched off with a hole pattern as shown in the right side of the figure. A tiny needle is placed on the top substrate with its pinhead hanging over the center of the bottom hole. When an ac voltage is applied between the ITO electrode and the needle, a radial electric field is generated within the hole. Subsequently, the LC molecules within the hole are reoriented in the radial direction. In this experiment, the hole diameter is $\sim 6 \mathrm{~mm}$, cell gap is $\sim 50 \mu \mathrm{m}$, and the substrates do not have any alignment layer. The $\mathrm{LC} /$ monomer mixture is comprised of $75 \mathrm{wt} \%$ Merck nematic LC E48 and $25 \mathrm{wt} \%$ diacrylate monomer RM82 (containing $\sim 1$ wt $\%$ photoinitiator IRG184). The mixture was uniformly mixed and injected into an empty LC cell in an isotropic phase. A voltage of $190 \mathrm{~V}_{\mathrm{rms}}$ was then applied to the cell. The cell was then slowly cooled down to room temperature and finally exposed to an UV light $\left(\lambda \sim 365 \mathrm{~nm}, I \sim 10 \mathrm{~mW} / \mathrm{cm}^{2}\right)$ to cure the LC gel for $30 \mathrm{~min}$. The cured monomers freeze the LC orientation direction even though the voltage is removed.

During cell fabrication, the radial electric field is present so that the LC directors are aligned along the radial direction. When a linearly polarized light is incident normally to the cell, the LC directors at different orientations will experience different phase retardations. For example, if the incident light passes through the center of the hole and its polarization is along the $y$ axis as Fig. 2 shows, then its exit polarization state would remain unchanged. However, if the incident linearly polarized light is deviated from the center of the hole, it will see two refractive indices: $n_{e}$ and $n_{o}$ are the extraordinary and ordinary refractive indices of the LC medium, respectively. The experienced phase retardation $(\delta)$ depends on the azimuthal angle $(\theta)$ as ${ }^{5}$

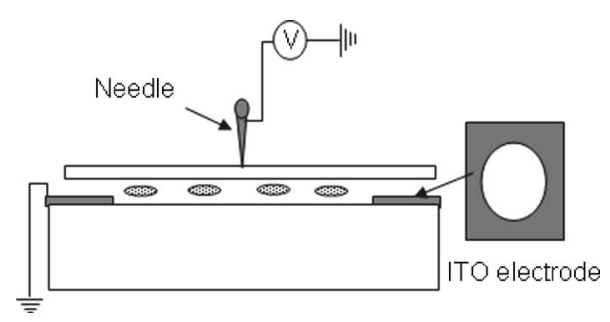

FIG. 1. Device structure of a radial cell. The aperture of the electrode hole is $\sim 6 \mathrm{~mm}$ and the LC cell gap is $\sim 50 \mu \mathrm{m}$. 


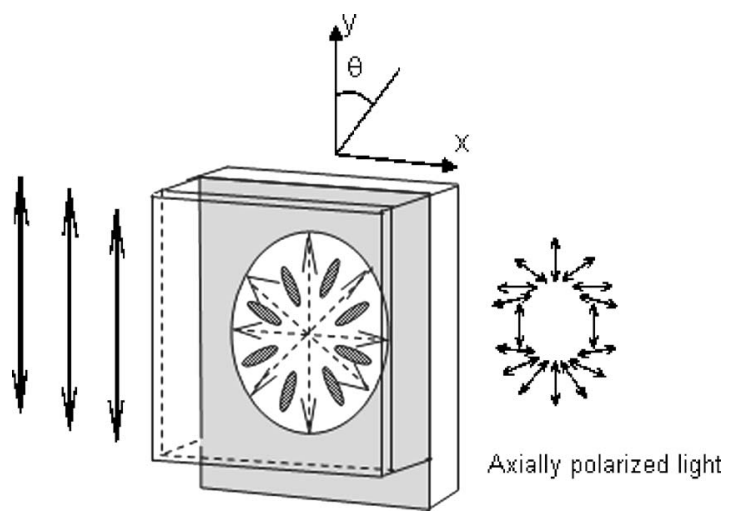

FIG. 2. Operation mechanism of the radial cell.

$$
\delta=\frac{2 \pi}{\lambda}(\Delta n)_{\mathrm{eff}} d,
$$

where

$$
(\Delta n)_{\mathrm{eff}}=\frac{n_{e} n_{o}}{\left[n_{e}^{2} \cos ^{2} \theta+n_{o}^{2} \sin ^{2} \theta\right]^{1 / 2}}-n_{o},
$$

$d$ is the LC cell gap, and $\lambda$ is the wavelength. From Eqs. (1) and (2), a different angle $\theta$ would lead to a different phase retardation. As the phase retardation changes, the output polarization state varies accordingly. As Fig. 2 shows, the principal axes of those polarized lights rotate continuously. If we observe the output light under crossed polarizers, then the region in which the LC directors are perpendicular to the analyzer should appear dark. Due to the radial symmetry of the LC gel, it is easy to obtain the two-dimensional intensity pattern when observed under crossed polarizers. In the special case of the radial cell designed to be a half-wave plate, i.e., the thickness of the LC layer satisfies the following equation:

$$
d=\frac{(2 m+1) \lambda}{2\left(n_{e}-n_{o}\right)}
$$

where $m$ is zero or a positive integer, then the LC molecules at azimuthal angle $\theta$ will rotate the output polarization by an angle of $2 \theta$ while still preserving the linear polarization. ${ }^{3}$

To confirm the above analysis, the intensity patterns of the LC cell were observed using a polarized optical microscope. Figure 3 shows the recorded intensity pattern between crossed polarizers. Indeed, the regions in which LC directors are parallel or perpendicular to the optical axis of the analyzer appear dark. The crosshair pattern remains unchanged

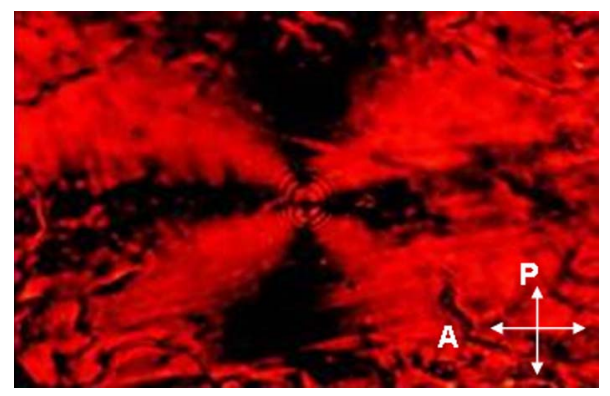

FIG. 3. (Color online) Recorded intensity patterns of a radial LC gel using a polarizing optical microscope. Polarizers are crossed. P: polarizer and A: analyzer.

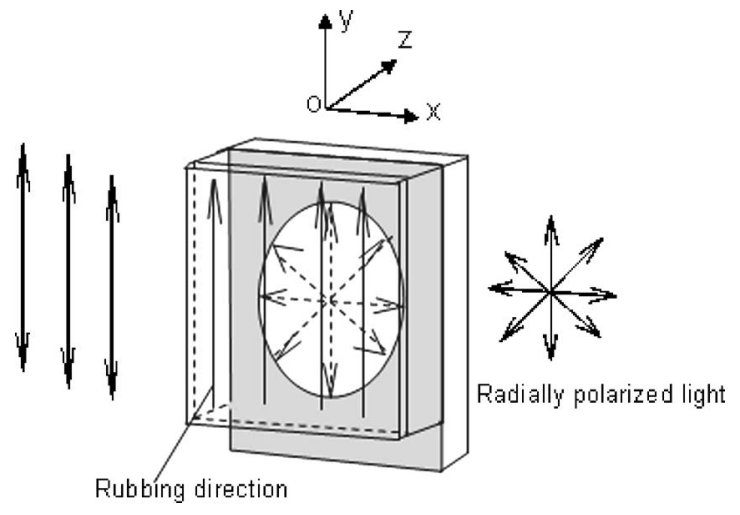

FIG. 4. Structure of a twisted-radial cell.

when we rotate the LC gel in the azimuthal direction. This confirms that the LC gel has radial orientation.

Another interesting structure is to use a top substrate that has a homogeneous rubbing alignment treatment while keeping the other parts, as shown in Fig. 1, unchanged. When the $\mathrm{LC} /$ monomer mixture was injected into the empty cell, the LC directors on the rubbed surface presented homogeneous alignment along the $y$ axis, as depicted in Fig. 4. If a radial electric field is applied in the hole-patterned region (same as Fig. 1), then the LC directors on the bottom substrate will present radial orientation. Along the $y$ axis and through the origin, the twist angle is $0^{\circ}$. But along the $x$ axis and through the origin, the twist angle is $90^{\circ}$. In the four quadrants, the twist angle of the LC directors would vary continuously from $0^{\circ}$ to $90^{\circ}$ or vice versa. When the dispersed monomer RM82 in the LC bulk is UV cured, the LC orientation structure is fixed. We called this structure a twisted-radial cell.

When a linearly polarized light is normally incident on the cell with its polarization direction parallel to the $y$ axis, the light polarization direction will follow the LC director orientation. In the yoz plane, the LC is not twisted so that the output polarization direction does not change. In the $x o z$ plane, though, the LC has a $90^{\circ}$ twist so that the output polarization direction will be twisted by $90^{\circ}$. From the yoz plane to the $x o z$ plane, the output polarization direction is twisted continuously. Therefore, the linearly polarized light became radially polarized, as depicted in Fig. 4.

When the LC cell was placed between crossed polarizers, a fan-shaped intensity pattern was observed. Figures 5 shows the intensity pattern observed from a polarizing optical microscope between crossed polarizers. If we rotate the

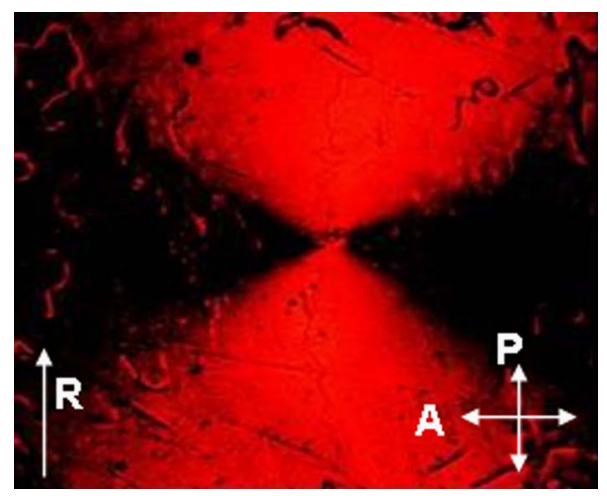

FIG. 5. (Color online) Recorded intensity patterns of a twisted-radial cell using a polarizing optical microscope. Polarizers are crossed. P: polarizer, A: analyzer, and R: rubbing direction. 
analyzer in the azimuthal direction, the axis of the fanshaped pattern would rotate synchronously. This is evidence of the axial LC orientation. Such experimental results agreed well with the depicted structure shown in Fig. 4. The twistedradial cell can convert a laser beam from linear to radial polarization or vice versa. Such a device can be used as a polarization axis finder or a versatile tool for investigating biological tissues and polarizing materials. The radial cell can also be used to design a tunable birefringent lens and a phase modulator.

If we replace the employed positive dielectric anisotropy $(\Delta \varepsilon)$ LC with a negative $\Delta \varepsilon$ LC in the cell structure as Fig. 4 shows, then the LC directors will present concentrically circular orientation induced by a radial electric field. The LC directors will exhibit the same structure as that induced by circularly rubbing the substrate surface. ${ }^{4}$ Depending on the polarization direction of the input light, such a LC cell can generate radially or azimuthally polarized light.

Depending on the cell structure and the LC material employed (positive or negative $\Delta \varepsilon$ ), various polarization converters based on the proposed LC gel can be fabricated. The major advantages of these devices are simple fabrication, low cost, and large size capability. To obtain a large aperture polarization converter, a high voltage is needed during the cell fabrication process. Similar to the LC microlens array, ${ }^{6,7}$ the ITO electrode in the bottom substrate can be etched into hole-array patterns. Using a needle array aligned with the bottom electrode-hole array, a micropolarization converter array can be fabricated using our approach, which otherwise would be difficult to achieve.

In conclusion, radial and twisted-radial cells based on LC gels are demonstrated. The radial cell can convert linearly polarized light to axially polarized light while the twisted-radial cell can convert linearly polarized light to radially polarized light. Based on our elementary approach, various polarization converters can be fabricated. In addition to a single large aperture, a microarray polarization converter can also be created. Such converters have potential applications in phase modulation, polarization-compensating system, interferometric techniques, and diffractive optical elements.

The authors would like to thanks H. Xianyu, S. Gauza, and $\mathrm{R}$. Lu for their kind help during experimentation and their useful discussions, and Benjamin Wu for proofreading this letter.

${ }^{1}$ M. Stalder and M. Schadt, Opt. Lett. 21, 1948 (1996).

${ }^{2}$ J. A. Davis, D. E. McNamara, D. M. Cottrell, and T. Sonehara, Appl. Opt. 39, 1549 (2000).

${ }^{3}$ Y. H. Wu, Y. H. Lin, H. Ren, X. Nie, J. H. Lee, and S. T. Wu, Opt. Express 13, 4638 (2005)

${ }^{4}$ R. Yamaguchi, T. Nose, and S. Sato, Jpn. J. Appl. Phys., Part 1 28, 1730 (1989).

${ }^{5}$ S. T. Wu and D. K. Yang, Reflective Liquid Crystal Displays (Wiley, New York, 2001).

${ }^{6}$ T. Nose, S. Masuda, S. Sato, J. Li, L. C. Chien, and P. J. Bos, Opt. Lett. 22, 351 (1997).

${ }^{7}$ H. Ren, Y. H. Lin, and S. T. Wu, Opt. Commun. 261, 296 (2006). 